\title{
Application of queuing theory in production-inventory optimization
}

\author{
Reza Rashid $^{1} \cdot$ Seyed Farzad Hoseini ${ }^{1}$ - M. R. Gholamian ${ }^{1} \cdot$ Mohammad Feizabadi $^{2}$
}

Received: 4 December 2014 / Accepted: 29 May 2015/Published online: 4 July 2015

(c) The Author(s) 2015. This article is published with open access at Springerlink.com

\begin{abstract}
This paper presents a mathematical model for an inventory control system in which customers' demands and suppliers' service time are considered as stochastic parameters. The proposed problem is solved through queuing theory for a single item. In this case, transitional probabilities are calculated in steady state. Afterward, the model is extended to the case of multi-item inventory systems. Then, to deal with the complexity of this problem, a new heuristic algorithm is developed. Finally, the presented bi-level inventory-queuing model is implemented as a case study in Electroestil Company.
\end{abstract}

Keywords Production inventory - Queuing theory · Multi-item inventory $\cdot$ Heuristic algorithm

\section{Introduction}

Nowadays, supply chains play an important role to meet diverse needs of customers. A supply chain can be defined as a network of organizations that collaborate to control and manage materials and information flow from suppliers to customers (Aitken 1998). One of the challenging issues in supply chain management is to find optimal policies for inventory systems. The main objective of inventory management is to balance conflicting goals such as stock costs and shortage costs (Arda and Hennet 2006).

Seyed Farzad Hoseini

f_hoseini@ind.iust.ac.ir

1 Department of Industrial Engineering, Iran University of Science and Technology, Narmak, 16844 Tehran, Iran

2 Department of Industrial Engineering, Sharif University, Azady, Tehran, Iran
Day by day, the number of researchers, attracted to production-inventory systems in supply chain, is increasing. In inventory management point of view, a manufacturer with a limited production capacity needs to hold finished goods inventory as safety stocks. For this inventory system, if demand is less than service capacity, the manufacturer needs to use different policies for different inventory levels.

For instance, it should use two points of inventory level: one for starting and the other for finishing the production line. For better description of the model, Fig. 1 illustrates the inventory level for the system when production rate $\mu$ and demand rate $D$ are considered deterministic. Actually, in a real-world system, different stochastic parameters may affect the model and increase the complexity of the system.

In summary, it is clear that in spite of many contributions to the stock control, there is little consideration regarding production-inventory models in stochastic environments. For this reason, we represent a mathematical model in which the main contributions of this paper can be summarized as follows:

- A production inventory system is developed in an uncertain environment.

- Queuing theory is used to provide a stochastic model.

- The proposed model is extended for multi-item inventory systems.

- A new heuristic algorithm is proposed to solve the model.

- All the steady-state equations are solved in one state.

This model can be probably applied to inventory systems where demand and production time are uncertain. Company has setup costs and it also holds finish goods inventory.

The remainder of this paper is organized as follows. In Sect. 2, a brief literature review is presented. In Sect. 3, an 


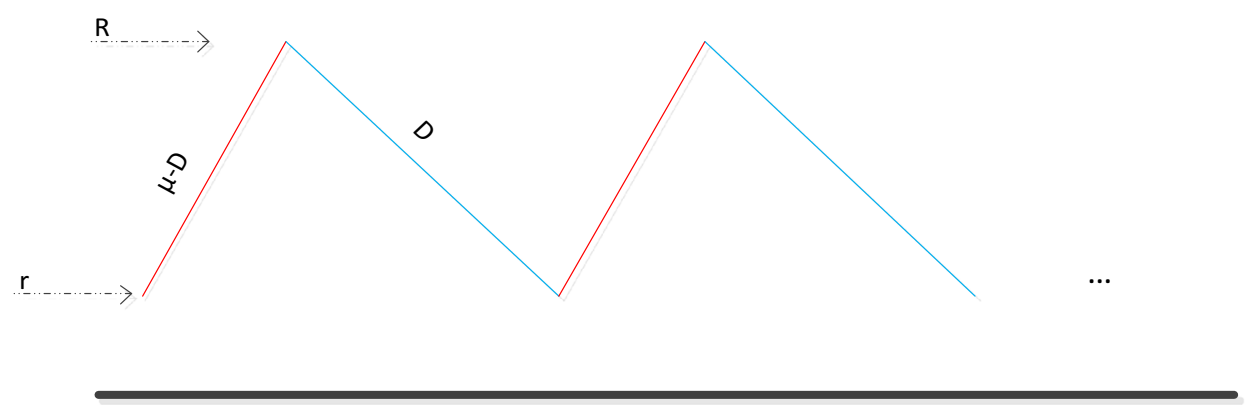

Fig. 1 Inventory level of the production model

inventory model is presented and then a branch and bound algorithm is proposed. Afterward, the model is validated in Sect. 4. Then, conclusions are made in Sect. 5.

\section{Literature review}

In the literature, there are some researchers who have dealt with production-inventory systems. For example, Yu and Dong (2014) considered a two-stage production lot sizing problem which used an inventory system with random demand arrivals. To solve the problem, they proposed a numerical approach for the problem. Baek and Moon (2014) considered a lost sales production-inventory system in an uncertain environment. They also used queuing theory to present a stochastic model for the system. Also, some production-inventory papers in the literature assumed that the lead time is negligible or it can be ignored in practice when it is short in contrast to other time factors (Karimi-Nasab and Seyedhoseini 2013). Most of such research papers did not consider lost sales. Many of them, however, considered back orders as a rational managerial policy.

Chang and $\mathrm{Lu}$ (2011) considered a serial production system controlled by the base-stock policy. They presented a phase-type approximation for a controlled base-stock serial production system. They also proposed a cost model to determine the optimal base-stock level.

Jewkes and Alfa (2009) considered a production system in which a supplier produces semi-finished items on a make-to-stock basis for a manufacturer that customizes the items on a make-to-order basis. The manufacturer attempts to determine the optimal point of differentiation and its optimal semi-finished goods buffer size. They used matrix geometric methods to evaluate performance through various measures for this system.

Some researchers worked on multi-item inventory systems. For instance, Shavandi et al. (2012) proposed a new constrained multi-item pricing and inventory model. They covered three categories of perishable products in their model. Taleizadeh et al. (2012) dealt with a multi-product inventory control problem in which periods between two replenishments were assumed to be independent random variables.

Generally, in a stock control system, most of the parameters are not deterministic. Thus, some researchers used queuing theory to construct their models. In this area, there are some researchers who provided a model for stochastic demand. Some researchers presented a model for stochastic lead time. Parlar (1997) presented an inventory model which was combined with queuing theory to consider demand and lead time as stochastic parameters. Ha (1997) considered Poisson distribution for demands and exponential for production times in a single item make-tostock production system. He proposed an $M / M / 1 / S$ queuing system for modeling the system. Arda and Hennet (2006) addressed inventory control of a multi-supplier strategy in a two-level supply chain. They considered random arrivals for customers and random delivery time for suppliers and represented the system as a queuing network.

Sapna Isotupa (2006) considered lost sales of $(s, Q)$ inventory system with two customer groups and illustrated the Markov processes. Boute et al. (2007), in a two-echelon supply chain, show that by including the impact of the order decision on lead times, the order pattern can be smoothed to a considerable extent without increasing stock levels. Karimi-Nasab and Konstantaras (2013) considered special sales offer for a single item from the supplier under stochastic replenishment intervals.

Jain and Raghavan (2009) studied batch ordering in multi-echelon supply chains and used queuing theory to capture the behavior of the manufacturing supply chain network. Babai et al. (2011) investigated stochastic demand and lead time and analyzed a single item inventory system through queuing theory. Bahri and Tarokh (2012) assumed that the delivery lead time is stochastic and follows an exponential distribution. Also, the shortage during the lead time is permitted and completely back ordered for the buyer.

Seyedhoseini et al. (2014) considered Poisson demand for customer in a cross docking problem. They employed queuing theory to provide a stochastic model. Salameh et al. 
(2014) combined the separate works on substitution and joint replenishment and introduced a solution procedure for solving the joint replenishment model with substitution for two products within the framework of the classical economic order quantity model. Karimi-Nasab and SabriLaghaie (2014) extended classical economic production quantity model to the case of stochastically generated poorquality items, while an imperfect screening scheme was devised to recognize such items from healthy ones.

Krommyda et al. (2015) studied an inventory control problem in which demand was satisfied using two mutually substitutable products. Their aim was to determine the order quantity for each product that maximizes the joint profit function.

Seyedhoseini et al. (2015) applied queuing theory to propose a mathematical model for inventory systems with substitute flexibility. (Rashid et al. 2015) also considered a location-inventory model. To prepare a stochastic inventory model, they used bi-level Markov process. Considering the effectiveness of queuing theory in inventory problems, we have also used queuing theory to develop a stochastic stock control model.

As demonstrated in Table 1, there are some researchers who considered $(R, Q)$ policy in stochastic programming. However, some of them did not consider multi-item inventory, and some of them did not consider production inventory. Also, few researchers have analyzed their model with queuing theory. Hence, we propose a mathematical model for the production-inventory problem in the stochastic environment.

\section{Model development}

In this section, first, the proposed model is developed. Then, it is extended for the multi-item production-inventory system. Afterward, a new algorithm is proposed to solve the model. As for notifications, parameters are described as follows:

\begin{tabular}{|c|c|c|c|}
\hline$r$ & Reorder level & $(I, J)$ & $\begin{array}{l}\text { State for } I \text { inventory and } \\
\text { being at mood of } J\end{array}$ \\
\hline$R$ & Optimal level & $\pi_{I, J}$ & $\begin{array}{l}\text { Steady-state probability } \\
\text { of state }(I, J)\end{array}$ \\
\hline$D$ & Demand & $S$ & Amount of shortage \\
\hline$\mu$ & Service rate & $M$ & Number of product types \\
\hline SE & Setup cost & $C_{i, I}$ & Holding costs \\
\hline$C_{i, S}$ & Shortage costs & $r_{i}$ & Reorder level for product $i$ \\
\hline$\pi_{r+1, D, i}$ & $\begin{array}{l}\text { Steady-state probability } \\
\text { of being in state }(r+1 \text {, } \\
D) \text { for product } i\end{array}$ & $R_{i}$ & Optimal level for product $i$ \\
\hline
\end{tabular}

\section{Model description}

In this paper, a historical production-inventory model is developed. Moreover, a new stochastic multi-item production-inventory model in which shortages have been considered lost sales is proposed. In this strategy, the production cycle starts when the manufacturer's inventory falls to or below the reorder point $r$ and stops when its onhand inventory reaches its optimal level $R$.

Inspired by Sapna Isotupa (2006) who developed a stochastic inventory model for $(R, Q)$ systems, we also developed a new stochastic model for the historical production-inventory model. Thus, the customers' arrival is assumed to be Poisson distribution with rate of $D$, and production time is negative exponentially distribution with rate of $\mu$. Also, it has been assumed that if one type of products is not under production, its production capacity would equally divide to the other products. To achieve a comprehensive model, queuing theory is employed to describe the inventory system. The transition diagram for this model is illustrated in Fig. 2.

For this diagram, states of the system have been decomposed into two sets: $A$ and $B$. In set $A$, the system is in production mode and in set $B$ system in demand mode; in demand mode, no production would occur. Also, states of the system are represented by $(I, J)$, where $I \in\{0,1, \ldots, R\}$ demonstrates the level of inventory and $J \in\{P, D\}$ demonstrates the mode of the system. Henceforth, let $\pi_{i, j}$ denote the steady-state probability of state ( $I$, $J)$. For this queue, three lemmas are proposed to set steadystate probabilities.

Lemma 1 Steady-state probabilities in terms of state $\pi_{R-1, P}$ for the system can be calculated as follows:

$$
\begin{aligned}
\pi_{R-2, P}= & \left(\frac{\mu+D}{\mu}\right) \times \pi_{R-1, P} \\
\pi_{R-l, P}= & \left(\sum_{j=0}^{l-3}\left(\frac{D}{\mu}\right)^{j}\right)+\left(\frac{D+\mu}{\mu}\right) \times\left(\frac{D}{\mu}\right)^{l-2} \times \pi_{R-1, P} \\
\forall l \in & (3, \ldots, R-r), \\
\pi_{r-k, P}= & \left(\frac{D}{\mu}\right)^{k} \times\left(\sum_{j=0}^{R-r-3}\left(\frac{D}{\mu}\right)^{j}\right) \\
& +\left(\left(\frac{D+\mu}{\mu}\right) \times\left(\frac{D}{\mu}\right)^{R-r-2}\right) \times \pi_{R-1, P} \\
\forall k \in & (1, \ldots, r), \quad \forall i \in(r+1, \ldots, R) . \\
\pi_{i, D}= & \frac{\mu}{D} \times \pi_{R-1, P} \quad \forall i \in
\end{aligned}
$$


Table 1 Literature of the stochastic inventory and production inventory

\begin{tabular}{|c|c|c|c|c|c|c|c|c|}
\hline \multirow[t]{2}{*}{ References } & \multicolumn{3}{|c|}{ Shortage } & \multirow[t]{2}{*}{ Lead time } & \multirow[t]{2}{*}{ Demand } & \multirow{2}{*}{$\begin{array}{l}\text { Replenishment } \\
\text { policy }\end{array}$} & \multirow{2}{*}{$\begin{array}{l}\text { Multi-item } \\
\text { inventory }\end{array}$} & \multirow{2}{*}{$\begin{array}{l}\text { Production } \\
\text { inventory }\end{array}$} \\
\hline & $\begin{array}{l}\text { Lost } \\
\text { sales }\end{array}$ & $\begin{array}{l}\text { Back } \\
\text { order }\end{array}$ & $\begin{array}{l}\text { Did not } \\
\text { consider }\end{array}$ & & & & & \\
\hline Sapna Isotupa (2006) & & $\sqrt{ }$ & & Stochastic & Stochastic & $(R, Q)$ & No & No \\
\hline Arda and Hennet (2006) & & $\sqrt{ }$ & & Stochastic & Stochastic & $(S-1, S)$ & No & Yes \\
\hline Boute et al. (2007) & & $\sqrt{ }$ & & Stochastic & Stochastic & $(T, S)$ & No & No \\
\hline Hill et al. (2007) & $\sqrt{ }$ & & & Deterministic & Stochastic & $(S-1, S)$ & No & No \\
\hline Hennet and Arda (2008) & & $\sqrt{ }$ & & Stochastic & Stochastic & $(S-1, S)$ & No & No \\
\hline Chang and Lu (2011) & $\sqrt{ }$ & $\sqrt{ }$ & & Stochastic & Stochastic & $(S-1, S)$ & No & Yes \\
\hline Babai et al. (2011) & & $\sqrt{ }$ & & Stochastic & Stochastic & $(S-1, S)$ & No & No \\
\hline Taleizadeh et al. (2012) & & $\sqrt{ }$ & & Stochastic & Dynamic & $(T, s, S)$ & Yes & No \\
\hline Tlili et al. (2012) & $\sqrt{ }$ & & & Deterministic & Stochastic & $(T, s, S)$ & No & No \\
\hline Bahri and Tarokh (2012) & & $\sqrt{ }$ & & Stochastic & Deterministic & $(R, Q)$ & No & Yes \\
\hline Shavandi et al. (2012) & & $\sqrt{ }$ & & Deterministic & Deterministic & $(T, s, S)$ & Yes & Yes \\
\hline Alimardani et al. (2013) & & $\sqrt{ }$ & & Stochastic & Stochastic & $(S-1, S)$ & Yes & No \\
\hline Guerrero et al. (2013) & & & $\sqrt{ }$ & Deterministic & Stochastic & $(T, s, S)$ & Yes & No \\
\hline Karimi-Nasab and Konstantaras (2013) & & $\sqrt{ }$ & & Stochastic & Deterministic & $(R, Q)$ & No & No \\
\hline Karimi-Nasab and Seyedhoseini (2013) & & $\sqrt{ }$ & & Deterministic & Deterministic & $(T, S)$ & Yes & Yes \\
\hline Karimi-Nasab and Sabri-Laghaie (2014) & & $\sqrt{ }$ & & Deterministic & Deterministic & $(R, Q)$ & No & Yes \\
\hline Salameh et al. (2014) & $\sqrt{ }$ & & & Deterministic & Deterministic & $(R, Q)$ & Yes & No \\
\hline Yu and Dong (2014) & & & $\sqrt{ }$ & Deterministic & Stochastic & $(R, Q)$ & No & Yes \\
\hline Rashid et al. (2015) & $\sqrt{ }$ & & & Stochastic & Stochastic & $(R, Q)$ & No & No \\
\hline Seyedhoseini et al. (2015) & & & $\sqrt{ }$ & Deterministic & Stochastic & $(R, Q)$ & No & No \\
\hline Krommyda et al. (2015) & $\sqrt{ }$ & & & Deterministic & Deterministic & $(R, Q)$ & Yes & No \\
\hline Our model & $\sqrt{ }$ & & & Stochastic & Deterministic & $(R, Q)$ & Yes & Yes \\
\hline
\end{tabular}

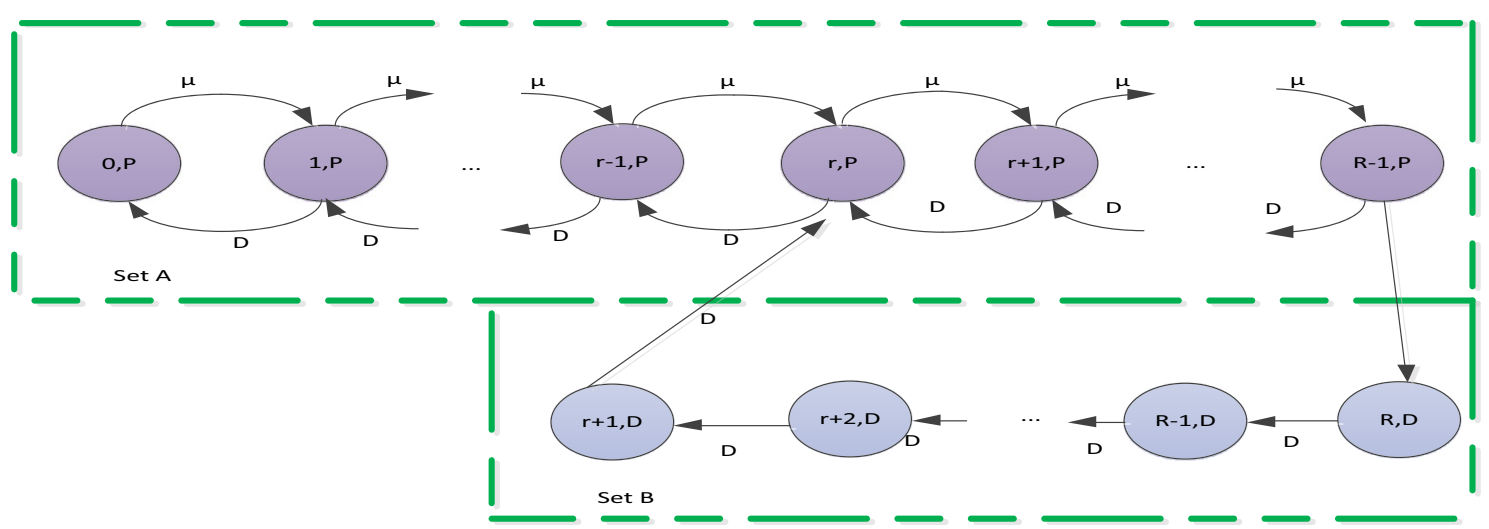

Fig. 2 Transition diagram for a product in the proposed model

Proof For a Markov process, Eq. 5 is true:

$\sum_{j=1}^{N} \lambda_{j x} \times \pi_{j}=\sum_{j=1}^{N} \lambda_{x j} \times \pi_{x}$

where $N$ is the number of all steady states, and $\lambda_{j x}$ is the probability rate from state $j$ to state $x$.
Regarding Fig. 1 and Eq. 5, Eq. 1 is true. Using Eq. 1, for $L$ between 3 and $R-r$, Eq. 6 could emerge, which proves Eq. 2,

$\mu \times \pi_{R-L, P}=D \times \pi_{R-L+1, P}+\mu \times \pi_{R-1, P}$.

When $I$ is smaller than $r$, then Eq. 7 is true: 
Fig. 3 Transition diagram for state $(0, p)$

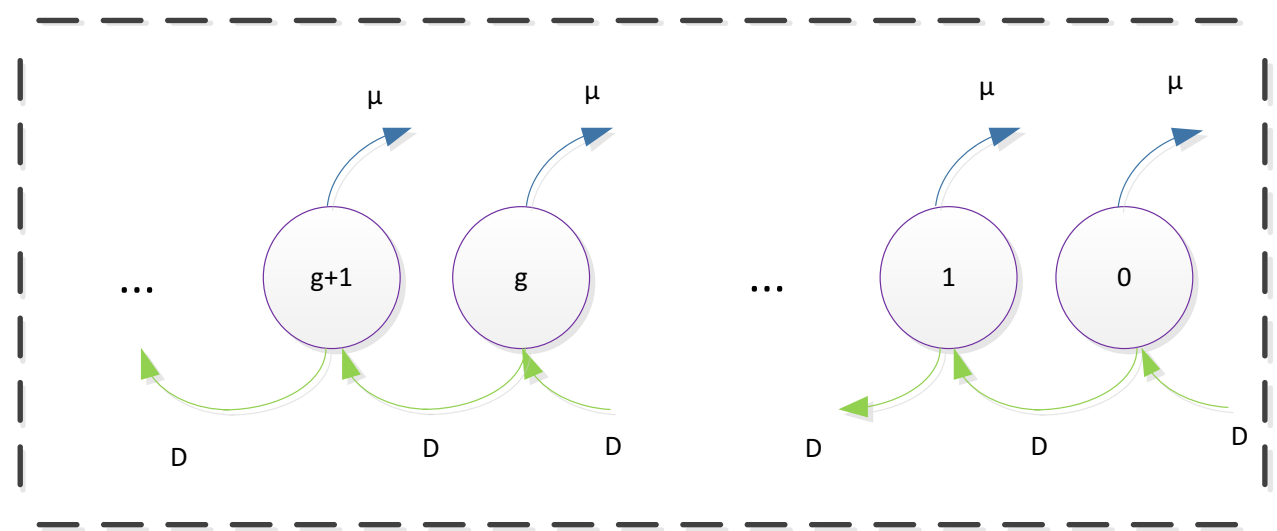

$\pi_{r-j, P}=\pi_{r, P} \times\left(\frac{D}{\mu}\right)^{j}$.

Using Eqs. 2 and 7 could result in Eq. 3 . As for set B, it is clear that the values of steady-state probabilities are equal and also Eq. 8 is true:

$\pi_{R, D}=\frac{\mu}{D} \times \pi_{R-1, P}$

So, Eq. 4 is true.Considering Lemma $1, \pi_{R-1, P}$ is equal to $\frac{1}{F}$, where $F$ can be calculated through Eq. 9:

$$
\begin{aligned}
F & =(R-r) \times \frac{\mu}{D}+1+\frac{\mu+D}{D}\left(1+\left(\frac{\frac{D}{\mu}-\left(\frac{D}{\mu}\right)^{R-r-1}}{1-\frac{D}{\mu}}\right)\right) \\
& +\frac{1}{1-\frac{D}{\mu}}\left((R-r-2)-\frac{\frac{D}{\mu}-\left(\frac{D}{\mu}\right)^{R-r-1}}{1-\frac{D}{\mu}}\right) \\
& +\left(\frac{\frac{D}{\mu}-\left(\frac{D}{\mu}\right)^{r+1}}{1-\frac{D}{\mu}}\right)\left(\frac{1-\left(\frac{D}{\mu}\right)^{R-r-2}}{1-\frac{D}{\mu}}+\frac{\mu+D}{D} \times\left(\frac{D}{\mu}\right)^{R-r-2}\right) .
\end{aligned}
$$

Lemma 2 The expected value for shortage can be computed through the following equation:

$S=\pi_{0} \times\left(\frac{D}{\mu}\right)$.

Proof The shortage occurs when the producer has no inventory and also a demand is announced to the retailer. In this section, $g$ is used for representing a state of shortage. For computing the expected value of shortage, we decompose state $(0, P)$ as in Fig. 3.
For this queue, if $\pi_{g}^{s}$ represents a steady-state probability of $g$ shortages, then it would be clear that:

$(D+\mu) \pi_{g+1}^{s}=(D) \pi_{g}^{s} \quad \forall g \geq 0$.

Considering Eq. 11, $\pi_{g}^{s}$ could be calculated as follows:

$\pi_{g}^{s}=\left(\frac{D}{D+\mu}\right)^{g} \pi_{0}^{s}$.

By considering Eq. 12, Eq. 13 could be obtained as

$\sum_{g=0}^{\infty}\left(\frac{D}{D+\mu}\right)^{g} \pi_{0}^{s}=\pi_{0}$.

Also, the average shortage is equal to:

$$
\begin{aligned}
S & =\pi_{0}^{s}\left(\left(\frac{D}{D+\mu}\right)+2\left(\frac{D}{D+\mu}\right)^{2}+\cdots+k\left(\frac{D}{D+\mu}\right)^{k}+\ldots\right) \\
& =\pi_{0}^{s} z\left(1+2(z)^{1}+\cdots+k(z)^{k-1}+\ldots\right),
\end{aligned}
$$

where $z$ is equal to $\frac{D}{D+\mu}$. In addition, it is known that:

$$
\begin{aligned}
(1 & \left.+2(z)^{1}+\cdots+k(z)^{k-1}+\ldots\right) \\
& =\frac{d}{d z}\left(z+(z)^{2}+\cdots+(z)^{k}+\ldots\right) \\
& =\frac{d}{d z}\left(\frac{z}{1-z}\right)=\frac{1}{(1-z)^{2}} .
\end{aligned}
$$

Considering Eqs. 14 and 15 could result in Eq. 10.

Lemma 3 For this inventory system, the expected value of the inventory level can be calculated by $A . \pi_{R-1, P}$, where A can be calculated by Eq. 16. 


$$
\begin{aligned}
& (R-1)+\frac{(R-2) \times \mu}{\mu+D}+(R-r) \times \frac{\mu}{D} \times\left(\frac{R+r+1}{2}\right)+R \times\left(\frac{\mu+D}{D}\right) \times\left(\frac{\frac{D}{\mu}-\left(\frac{D}{\mu}\right)^{R-r-1}}{1-\frac{D}{\mu}}\right) \\
& -\left(\frac{\mu}{D}\right) \times\left(\frac{\mu+D}{D}\right)\left[\frac{\left(1-(R-r+1) \times\left(\frac{D}{\mu}\right)^{R-r}\right)\left(1-\frac{D}{\mu}\right)+\frac{D}{\mu}-\left(\frac{D}{\mu}\right)^{R-r+1}}{\left(1-\frac{D}{\mu}\right)^{2}}-\left(1+2 \frac{D}{\mu}\right)\right] \\
& +R \times\left(\frac{1}{1-\frac{D}{\mu}}\right) \times\left[R-r-2-\left(\frac{\frac{D}{\mu}-\left(\frac{D}{\mu}\right)^{R-r-1}}{1-\frac{D}{\mu}}\right)\right]-\left(\frac{1}{1-\frac{D}{\mu}}\right) \times\left[\frac{(R-r+3)(R-r-2)}{2}\right] \\
& +\left(\frac{1}{1-\frac{D}{\mu}}\right) \times\left(\frac{\mu}{D}\right) \times\left[\frac{\left(1-(R-r+1) \times\left(\frac{D}{\mu}\right)^{R-r}\right)\left(1-\frac{D}{\mu}\right)+\frac{D}{\mu}-\left(\frac{D}{\mu}\right)^{R-r+1}}{\left(1-\frac{D}{\mu}\right)^{2}}-\left(1+2 \frac{D}{\mu}\right)\right] \\
& +\left(\frac{1-\left(\frac{D}{\mu}\right)^{R-r-2}}{1-\frac{D}{\mu}}+\frac{\mu+D}{D} \times\left(\frac{D}{\mu}\right)^{R-r-2}\right) \\
& {\left[r \times\left(\frac{\frac{D}{\mu}-\left(\frac{D}{\mu}\right)^{r+1}}{1-\frac{D}{\mu}}\right)-\frac{D}{\mu}\left(\frac{\left(1-(r+1) \times\left(\frac{D}{\mu}\right)^{r}\right)\left(1-\frac{D}{\mu}\right)+\frac{D}{\mu}-\left(\frac{D}{\mu}\right)^{r+1}}{\left(1-\frac{D}{\mu}\right)^{2}}\right)\right]}
\end{aligned}
$$

Proof For this inventory system, Eq. 17 is true:

$$
\begin{aligned}
\bar{I}= & \sum_{j=1}^{R-1} \pi_{j, P} \times j+\sum_{k=r+1}^{R} \pi_{k, D} \times k=\sum_{l=3}^{R-r}\left(\frac{\mu+D}{D}\right) \times\left(\frac{D}{\mu}\right)^{l-2} \\
& \times(R-l)+\sum_{l=3}^{R-r} \sum_{j=0}^{l-3}\left(\frac{D}{\mu}\right)^{j} \times(R-l) \\
& +\sum_{i-1}^{r}\left(\frac{D}{\mu}\right)^{i} \times\left(\sum_{j=0}^{R-r-3}\left(\frac{D}{\mu}\right)^{j}+\left(\frac{D+\mu}{\mu}\right) \times\left(\frac{D}{\mu}\right)^{R-r-2}\right) \\
& \times(r-i) .
\end{aligned}
$$

Also, for this queue the next three equations are true:

$$
\begin{aligned}
& \sum_{l=3}^{R-r}\left(\frac{\mu+D}{D}\right) \times\left(\frac{D}{\mu}\right)^{l-2} \times(R-l)=R \times\left(\frac{\mu+D}{D}\right) \\
& \times\left(\frac{\frac{D}{\mu}-\left(\frac{D}{\mu}\right)^{R-r-1}}{1-\frac{D}{\mu}}\right)-\left(\frac{\mu}{D}\right) \times\left(\frac{\mu+D}{D}\right) \\
& \quad \times\left[\frac{\left(1-(R-r+1) \times\left(\frac{D}{\mu}\right)^{R-r}\right)\left(1-\frac{D}{\mu}\right)+\frac{D}{\mu}-\left(\frac{D}{\mu}\right)^{R-r+1}}{\left(1-\frac{D}{\mu}\right)^{2}}\right. \\
& \left.-\left(1+2 \frac{D}{\mu}\right)\right],
\end{aligned}
$$




$$
\begin{aligned}
& \sum_{l=3}^{R-r} \sum_{j=0}^{l-3}\left(\frac{D}{\mu}\right)^{j} \times(R-l)=\sum_{l=3}^{R-r}\left(\frac{1-\left(\frac{D}{\mu}\right)^{l-2}}{1-\frac{D}{\mu}}\right) \times(R-l), \\
& =R \times\left(\frac{1}{1-\frac{D}{\mu}}\right) \times\left[R-r-2-\left(\frac{\frac{D}{\mu}-\left(\frac{D}{\mu}\right)^{R-r-1}}{1-\frac{D}{\mu}}\right)\right]-\left(\frac{1}{1-\frac{D}{\mu}}\right) \times\left[\frac{(R-r+3)(R-r-2)}{2}\right] \\
& +\left(\frac{1}{1-\frac{D}{\mu}}\right) \times\left(\frac{\mu}{D}\right) \times\left[\frac{\left(1-(R-r+1) \times\left(\frac{D}{\mu}\right)^{R-r}\right)\left(1-\frac{D}{\mu}\right)+\frac{D}{\mu}-\left(\frac{D}{\mu}\right)^{R-r+1}}{\left(1-\frac{D}{\mu}\right)^{2}}-\left(1+2 \frac{D}{\mu}\right)\right] \\
& \sum_{i-1}^{r}\left(\frac{D}{\mu}\right)^{i} \times\left(\sum_{j=0}^{R-r-3}\left(\frac{D}{\mu}\right)^{j}+\left(\frac{D+\mu}{\mu}\right) \times\left(\frac{D}{\mu}\right)^{R-r-2}\right) \times(r-i) \\
& \quad\left(\frac{1-\left(\frac{D}{\mu}\right)^{R-r-2}}{1-\frac{D}{\mu}}+\frac{\mu+D}{D} \times\left(\frac{D}{\mu}\right)^{R-r-2}\right)\left[r \times\left(\frac{\frac{D}{\mu}-\left(\frac{D}{\mu}\right)^{r+1}}{1-\frac{D}{\mu}}\right)-\frac{D}{\mu}\left(\frac{\left(1-(r+1) \times\left(\frac{D}{\mu}\right)^{r}\right)\left(1-\frac{D}{\mu}\right)+\frac{D}{\mu}-\left(\frac{D}{\mu}\right)^{r+1}}{\left(1-\frac{D}{\mu}\right)^{2}}\right)\right]
\end{aligned}
$$

Using Eqs. 17, 18, 19, and 20, Eq. 16 can emerge.

\section{Multi-product inventory model}

In this section, we considered multi-item inventory to determine near optimal $R_{i}, r_{i}$ and $\mu_{i}$ for each product. This objective is denoted through minimizing Eq. 21:

$\min z=\sum_{i=1}^{M}\left(\bar{I}_{i} \times C_{i, I}+\bar{S}_{i} \times C_{i, S}+\pi_{r+1, D, i} \times \mathrm{SE}_{i}\right)$.

For the inventory system, $M$ types of products are considered. To present our heuristic algorithm, it is assumed that on demand mood the product has a capacity (production rate) that can be equally divided into the other products. So, Eq. 20 could be used to approximate the service rate for the type $k$ product:

$\mu_{k}=\frac{\mu}{M}\left(1+\frac{\sum_{l \neq k}\left(1-p_{l}\right)}{\sum_{l \neq k}\left(p_{l}\right)}\right)$,

where $n$ represents the number of products and $p_{l}$ is equal to $\sum_{i=0}^{R-1} \pi_{i, P, l}$, which represents the probability of being in production mood for product $l$. It can be calculated from Eq. 23. In Eq. 22, $\sum_{l \neq k}\left(1-p_{l}\right)$ approximates the number of products in the demand mood and $\sum_{l \neq k}\left(p_{l}\right)$ approximates the number of products in the production mood.

$$
\begin{aligned}
& {\left[1+\left(\frac{\mu_{l}}{\mu_{l}+D}\right)+\sum_{l=3}^{R-r} \sum_{j=0}^{l-3}\left(\frac{D}{\mu_{l}}\right)^{j}+\left(\frac{D+\mu_{i}}{\mu_{l}}\right) \times\left(\frac{D}{\mu_{l}}\right)^{l-2}\right.} \\
& \left.+\sum_{i=1}^{r}\left(\frac{D}{\mu_{l}}\right)^{i} \times \sum_{j=0}^{R-r-3}\left(\frac{D}{\mu_{l}}\right)^{j}+\left(\frac{D+\mu_{l}}{\mu_{l}}\right) \times\left(\frac{D}{\mu_{l}}\right)^{l-2}\right] \times \pi_{R-1, P} .
\end{aligned}
$$

Figure 4 illustrates the inventory level, rate of production and rate of demand for the two products.

In this study, $\mu_{i}$ is considered to be bigger than $D_{i}$. For fixed values of different variables, it is clear that increase in $R_{i}$ causes an increase in $\bar{I}_{i}$. Furthermore, it causes $\pi_{r+1, D, i}$ and $\bar{S}_{i}$ to be decreased. So, for fixed values of different variables, the long-run expected cost is pseudo-convex in $R$.

On the other hand, for fixed values of different variables, increase in $r_{i}$ would increase the probabilities of states which have more than $r_{i}$ inventory level, and it causes $\pi_{r+1, D, i}$ and $\bar{I}_{i}$ to be increased. Moreover, it causes a decrease in $\bar{S}_{i}$. Consequently, for a fixed $R$, the long-run expected cost is pseudo-convex in $r_{i}$.

\section{Heuristic algorithm}

Regarding the complexity of our model, simple models would take big computational times. For this reason, we proposed a two-phased algorithm. 
Fig. 4 Illustration of the inventory level for two products

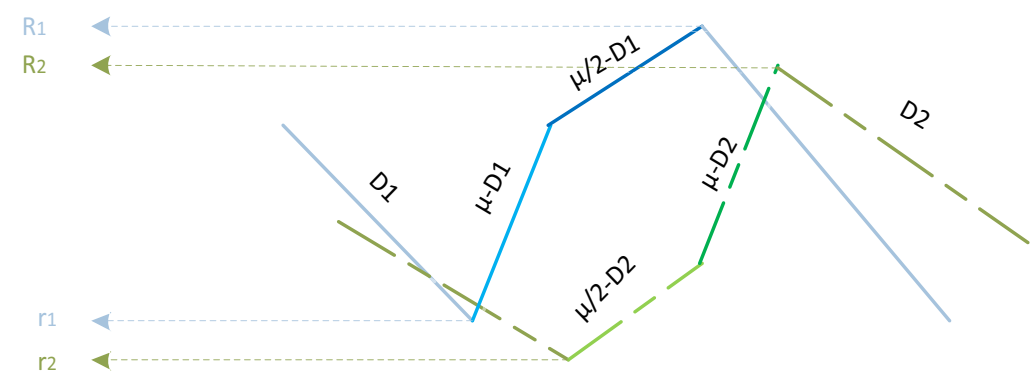

\section{Phase one}

In continuous space, $z$ is convex due to $r_{i}$ and $R_{i}$. We suppose $r_{i}$ and $R_{i}$ to be continuous and optimal and these were calculated by the steepest ascent method. For better description, it is assumed that there is only one product and if $r_{l i}$ and $\mathrm{R}_{\mathrm{li}}$ denote the solution of the steepest ascent method, then Fig. 5 demonstrates the optimal solution in discrete space for just one product.

Considering Fig. 5, for the discrete problem, the nearest points in any direction to the optimal solution could be found in a space in which $r_{i}$ is $\left|r_{l i}\right|$ or $\left|r_{l i}\right|+1$, and $R_{i}$ is $\left|R_{l i}\right|$ or $\left|R_{l i}\right|+1$. Consequently, a search among these nodes can provide a perfect local solution. Assuming a number of product equal to $M$, there would be $2^{2 M}$ nodes in the space that needs to be searched. So, for big $M$, phase two is proposed.

\section{Phase two}

Considering the optimal solution resulting from the steepest deepest algorithm, we proceed with the following steps:

Step 1 Calculate $\mu_{i}$ for each of the products for the continuous solution.

Step 2 For each product, search the nodes where $\left|r_{l i}\right|$ or $\left|r_{l i}\right|+1$, and $R_{i}$ is $\left|R_{l i}\right|$ or $\left|R_{l i}\right|+1$, and then calculate Eq. 24, independently,

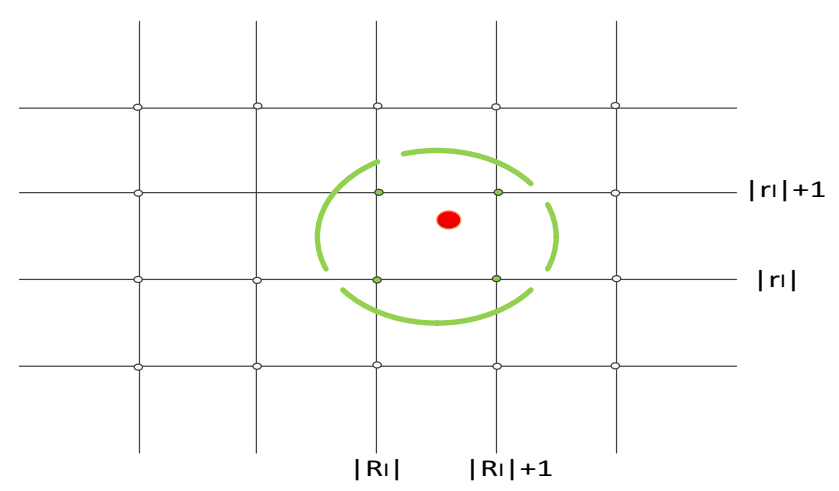

Fig. 5 Solution of the discrete problem

$$
z_{i}=\left(\bar{I}_{i} \times C_{i, I}+\bar{S}_{i} \times C_{i, S}+\pi_{r+1, D, i} \times \mathrm{SE}_{i}\right) .
$$

Step 3 Choose the nodes which have the minimum $z_{i}$ for each product.

\section{Computational results}

In this research, the proposed algorithm is coded in $\mathrm{C}++$ software. For evaluation of the algorithm, we produced five examples for $M$ equal to 10 , where all parameters are selected randomly while creating the data set. The parameters are set as follows: $C_{i, I} \in[1,10], C_{i, S} \in[1,10]$, $D_{i} \in[1,10], \mu=10 M$ and $S E_{i} \in[30,60]$. In this case, instead of letting $R$ go to infinity, the maximum value of $R$ was set to be 10,000 . Therefore, the maximum value of $s$ was 9999 . We also limited the computational time to $5 \mathrm{~h}$ and mean solving time for our algorithm was $223.097 \mathrm{~s}$, while none of the examples could be solved through searching all feasible solutions.

In this model, an example is generated to analyze the parameters of the model in which $C_{I}, C_{S}, D, \mathrm{SE}$ and $\mu$ are equal to $5,20,3,200$ and 4 , respectively. Also, the behavior of inventory costs has been studied for different values of $R$ and $r$ in Fig. 6.

It is proposed to use this model for companies using workshop system and reorder point for replenishing their finished goods inventory. To provide a better description of the model, an example is presented in Table 2.

A near optimal solution for this example is 164 where $\left(r_{1}, r_{2}, r_{3}, R_{1}, R_{2}, R_{3}\right)$ is equal to $(1,1,1,4,3,3)$, respectively. If each product is to be planned separately, the optimal cost would be equal to $211,28 \%$ more than our model's costs. We considered Product 3 and analyzed costs related to $D$ in Fig. 7. To analyze the performance of our heuristic, $R$ and $Q$ are limited to be less than 100, and the optimal solution for different $D$ s are prepared.

As in Fig. 7, the same pattern has been demonstrated for shortage and holding costs. On the other hand, the pattern of setup cost is totally different. Furthermore, there is not a great difference among heuristic and optimal costs. 


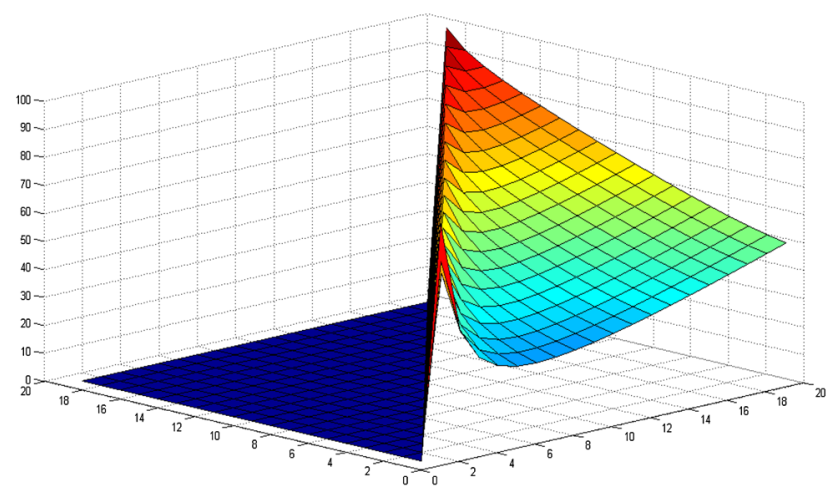

Fig. 6 Behavior of the model regarding $r$ and $R(\boldsymbol{r}<\boldsymbol{R})$

Table 2 An example for describing the proposed model

\begin{tabular}{llllr}
\hline$\mu=15$ & $C_{I}$ & $C_{\mathrm{s}}$ & $D$ & $\mathrm{SE}$ \\
\hline Product 1 & 10 & 100 & 4.5 & 50 \\
Product 2 & 20 & 250 & 4 & 70 \\
Product 3 & 30 & 300 & 4 & 100 \\
\hline
\end{tabular}
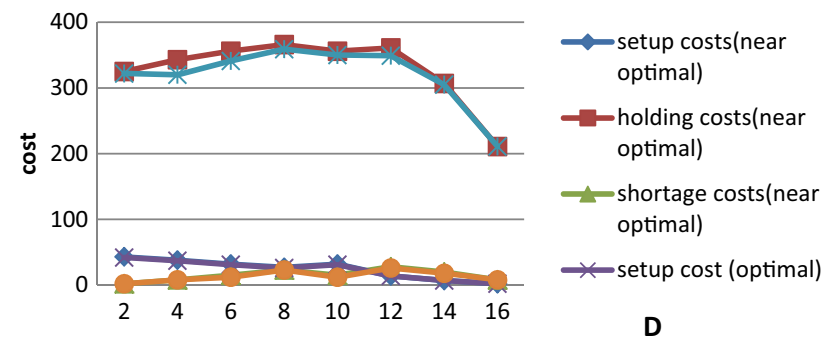

Fig. 7 Sensitivity of different costs due to the demand rate

This model could be implemented properly for workshop systems. The Electroestil Company was established in 1980 and now it is one of the most popular brands of refrigerator production in Iran. Electroestil produces different kinds of refrigerators for houses and shops. It has 20 types of refrigerators for houses and 15 types for shops.

In this company, there are four workshops and 1500 employees. This company has a production capacity of 800 products per day for house refrigerators and 120 products per day for shop refrigerators. Experts in Electroestil approximate that demands of house refrigerators for different product types are equal and they are equal to 30 per

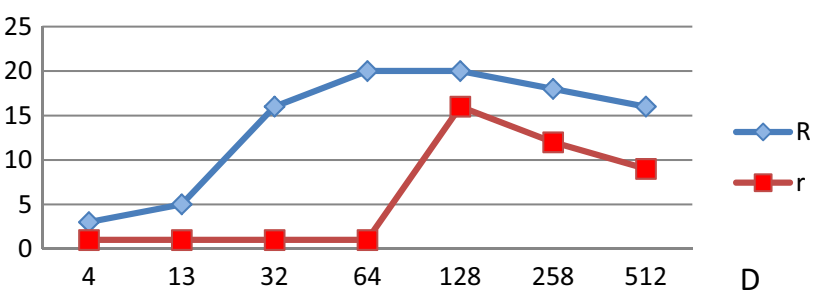

Fig. 8 Relation between $R, r$ and $D$ for ESR320

day, and demands of shop refrigerators for different product types are equal and they are equal to 7 per day. They have defined $C_{I}$ to be equal to 100,000 tomans for each product, $C_{S}$ to 2 million tomans-Iran's currency-for each product, and SE to 300,000 tomans for each product. For this company, Table 3 illustrates the performance of the proposed model. Significant changes in inventory costs have been determined if the proposed model is used.

As shown in this table, there is a big difference between the optimal solution and the current situation of the company. In the current situation, shortage costs and setup costs are small, but holding costs are big. For this company, the rate of production is much more than the rate of demand. This could be a reason for the low quantity of $r$.

In this model, the demand rate has significant effects on $r$ and $R$. Therefore, we only considered ESR320 which is a shop refrigerator. For this product, $R$ and $r$ were analyzed according to the demand rate in Fig. 7. The same pattern was displayed for them. Also, it has been discovered that as the demand rate becomes bigger, when it is bigger than the service rate, the optimal costs decrease. For example, the costs for $D=64$ is equal to 30 which is significantly less than for $D=7$. However, for small demand rates, its increase makes optimal costs bigger. For example, in $D=13$, the optimal cost is equal to 302 .

\section{Conclusion and future directions}

In this paper, a new production-inventory model is developed (Fig. 8). To prepare a cohesive model, demand and production time are considered as stochastic parameters and queuing theory is used to calculate long-run inventory costs. The proposed model also has been extended for multi-item inventory systems. The inventory model has

Table 3 Comparison between our model and the current planning for each product $(\times 1000$ tomans)

\begin{tabular}{|c|c|c|c|c|c|c|c|c|c|c|}
\hline & \multicolumn{5}{|c|}{ House refrigerator } & \multicolumn{5}{|c|}{ Shop refrigerator } \\
\hline & $\bar{r}$ & $R$ & Inventory holding costs & Shortage costs & Setup costs & $r$ & $R$ & Inventory costs & Shortage costs & Setup costs \\
\hline Multi-item model & 1 & 4 & 175 & 82 & 41 & 1 & 3 & 144 & 49 & 73 \\
\hline Current planning & 8 & 20 & 1229.1 & 0.001 & 8.638 & 5 & 12 & 610.63 & 44.010 & 9.907 \\
\hline
\end{tabular}


been analyzed concerning $r$ and $R$. These parameter behaviors are investigated. We also proposed a heuristic algorithm to solve the problem. The results demonstrate the efficiency of the proposed algorithm. To prepare a better description of the model, a real-world example is studied and analyzed. Respecting the collected data, it has been observed that there is a significant relation between the demand rate and production rate. If demand is smaller than service rate, increase of demand causes an increase in optimal costs. On the other hand, when it is bigger than the service rate, a small increase may reduce the optimal costs.

Lost sales shortage may be considered as one of the weaknesses in our model. So, in future, the model can be extended through considering back-order shortage. This may increase the complexity of the problem while the model would become more realistic. Another extension of this research is possible by covering corruption rates for perishable inventories.

Open Access This article is distributed under the terms of the Creative Commons Attribution 4.0 International License (http:// creativecommons.org/licenses/by/4.0/), which permits unrestricted use, distribution, and reproduction in any medium, provided you give appropriate credit to the original author(s) and the source, provide a link to the Creative Commons license, and indicate if changes were made.

\section{References}

Aitken JM (1998) Supply chain integration within the context of a supplier association: case studies of four supplier associations. Cranfield University, UK

Alimardani M, Jolai F, Rafiei H (2013) Bi-product inventory planning in a three-echelon supply chain with backordering, Poisson demand, and limited warehouse space. J Ind Eng Int 9:22

Arda Y, Hennet J-C (2006) Inventory control in a multi-supplier system. Int J Prod Econ 104:249-259

Babai MZ, Jemai Z, Dallery Y (2011) Analysis of order-up-to-level inventory systems with compound Poisson demand. Eur J Oper Res 210:552-558

Baek JW, Moon SK (2014) The M/M/1 queue with a productioninventory system and lost sales. Appl Math Comput 233:534-544

Bahri M, Tarokh MJ (2012) A seller-buyer supply chain model with exponential distribution lead time. J Ind Eng Int 8:1-7

Boute RN, Disney SM, Lambrecht MR, Van Houdt B (2007) An integrated production and inventory model to dampen upstream demand variability in the supply chain. Eur J Oper Res 178:121-142

Chang K-H, Lu Y-S (2011) Inventory management in a base-stock controlled serial production system with finite storage space. Math Comput Model 54:2750-2759

Guerrero W, Yeung T, Guéret C (2013) Joint-optimization of inventory policies on a multi-product multi-echelon pharmaceutical system with batching and ordering constraints. Eur J Oper Res 231:98-108

Ha AY (1997) Stock-rationing policy for a make-to-stock production system with two priority classes and backordering. Naval Res Logist 44:457-472

Hennet J-C, Arda Y (2008) Supply chain coordination: a game-theory approach. Eng Appl Artif Intell 21:399-405

Hill RM, Seifbarghy M, Smith DK (2007) A two-echelon inventory model with lost sales. Eur J Oper Res 69:753-766

Jain S, Raghavan NS (2009) A queuing approach for inventory planning with batch ordering in multi-echelon supply chains. Central Eur J Oper Res 17:95-110

Jewkes EM, Alfa AS (2009) A queueing model of delayed product differentiation. Eur J Oper Res 199:734-743

Karimi-Nasab M, Konstantaras I (2013) An inventory control model with stochastic review interval and special sale offer. Eur J Oper Res 227:81-87

Karimi-Nasab M, Sabri-Laghaie K (2014) Developing approximate algorithms for EPQ problem with process compressibility and random error in production/inspection. Int $\mathrm{J}$ Prod Res 52:2388-2421

Karimi-Nasab M, Seyedhoseini S (2013) Multi-level lot sizing and job shop scheduling with compressible process times: a cutting plane approach. Eur J Oper Res 231:598-616

Krommyda I, Skouri K, Konstantaras I (2015) Optimal ordering quantities for substitutable products with stock-dependent demand. Appl Math Model 39:147-164

Parlar M (1997) Continuous-review inventory problem with random supply interruptions. Eur J Oper Res 99:366-385

Rashid R, Bozorgi-Amiri A, Seyedhoseini S (2015) Developing a new stochastic competitive model regarding inventory and price. J Ind Eng Int, pp 1-10

Salameh MK, Yassine AA, Maddah B, Ghaddar L (2014) Joint replenishment model with substitution. Appl Math Model 38: 3662-3671

Sapna Isotupa K (2006) An (s, Q) Markovian inventory system with lost sales and two demand classes. Math Comput Model 43: 687-694

Seyedhoseini S, Rashid R, Teimoury E (2014) Developing a crossdocking network design model under uncertain environment. J Ind Eng Int, pp 1-12

Seyedhoseini S, Rashid R, Kamalpour I, Zangeneh E (2015) Application of queuing theory in inventory systems with substitution flexibility. J Ind Eng Int, pp 1-8

Shavandi H, Mahlooji H, Nosratian NE (2012) A constrained multiproduct pricing and inventory control problem. Appl Soft Comput 12:2454-2461

Taleizadeh AA, Niaki STA, Seyedjavadi SMH (2012) Multi-product multi-chance-constraint stochastic inventory control problem with dynamic demand and partial back-ordering: a harmony search algorithm. J Manuf Syst 31:204-213

Tlili M, Moalla M, Campagne J-P (2012) The trans-shipment problem in a two-echelon, multi-location inventory system with lost sales. Int J Prod Res 50:3547-3559

Yu AJ, Dong Y (2014) A numerical solution for a two-stage production and inventory system with random demand arrivals. Comput Oper Res 44:13-21 